\title{
EXPERIENCIA DE CONSERVACIÓN DE CAÑA DE AZÚCAR PICADA CON AGREGADO DE CAL (OXIDO DE CALCIO) A TRAVÉS DE UN PRODUCTOR DEMOSTRADOR
}

\section{Chopped Sugar Cane with calcium oxide preservation experience performed by a role model producer}

\author{
Castelán, María E.; Porta, Miriam; Hack, Claudina M.; Burgos, Angela M. y Yanissek, Yonathan Y. \\ ${ }^{1}$ Instituto Agrotécnico "Pedro M. Fuentes Godo" - FCA-UNNE. ${ }^{2}$ Cátedra de Cultivos III - FCA- \\ UNNE. E-mail: castelanme@hotmail.com
}

\begin{abstract}
RESUMEN
La actividad ganadera tiene gran trascendencia para la Provincia de Corrientes dado que ocupa el 93\% del total de la superficie agropecuaria. Las pequeñas unidades productivas familiares utilizan los animales para la venta, como fuerza de trabajo, autoconsumo y para generar ingresos a través de la elaboración de quesos denominados criollos Teniendo en consideración los materiales forrajeros regionales de alta disponibilidad y capaces de aportar nutrientes a la dieta animal a bajo costo, se optó por la metodología de Productor Demostrador, con el objetivo de difundir la experiencia y activar el proceso de adopción de la técnica propuesta. La caña de azúcar tradicionalmente se corta, pica y se entrega diariamente al animal. Sin embargo, en grandes cantidades, esta técnica se convierte en un obstáculo para el uso porque requiere de mano de obra diaria para los cortes, molienda y transporte. La caña de azúcar mezclada con cal (óxido de Calcio) es un método de conservación, que en esta experiencia permitió almacenar el alimento durante 7 días. Se trabajó con un productor demostrador de la localidad de Santa Ana, Corrientes, cuya apreciación de la experiencia fue sumamente positiva. El mismo se mostró satisfecho ya que no solo incrementó la producción de leche diaria sino que también pudo organizar mejor el tiempo dedicado a la alimentación de los animales. Por ello, podrá enseñarles a sus pares esta técnica que ha podido validar, con lo que se espera la difusión y transferencia tenga mayor impacto.
\end{abstract}

Palabras clave: caña de azúcar, cal, conservación, demostrador.

\begin{abstract}
Cattle breeding is of great importance in the province of Corrientes since it occupies $93 \%$ of the total agricultural area. Small family productive units use cattle for trading, as draught animal power source, for self-supply and as revenue increase through the production of "criollo" cheese. Taking into account the great availability of regional forage material able to provide nutrients at low cost to the animal's diet, the role model producer's methodology was chosen with the aim of disseminating the experience and fostering acquisition of the proposed technique. Sugar cane is traditionally cut, chopped and delivered daily to the cattle. However, in large quantities, this technique becomes an obstacle because it requires daily handlabour for cutting, milling and transporting. Mixing up sugar cane with lime (Calcium oxide) is a preservation method, which in this experience allowed food supplies to be stored for up to 7 days. A livestock farmer with a role model from Santa Ana, Corrientes was summed to the experience, his appreciation was valuable to the experience. He was pleased because this technique did not only increase the daily production of milk, but also helped him to organise better the amount of time devoted to cattle feeding. Consequently, he will be able to transmit this already validated method to his colleagues, that it is hoped to be disseminated on order to produce great impact.Keywords: sugar cane, lime, preservation, demo producer.
\end{abstract}

Keywords: sugar cane, lime, preservation, demo producer.

Recibido: 17/jun/2020. Aceptado: 22/Mar/2021 


\section{INTRODUCCIÓN}

La actividad ganadera tiene gran trascendencia para la Provincia de Corrientes dado que ocupa el 93\% del total de la superficie agropecuaria. Existen 25.603 productores ganaderos de los cuales el $42 \%$ posee menos de 20 cabezas de ganado bovino (Acosta et al., 2009, SENASA, 2011). Las pequeñas unidades productivas familiares utilizan los animales como fuerza de trabajo, autoconsumo y para generar ingresos a través de la elaboración de quesos denominados criollos. La elaboración de quesos artesanales conocidos como "criollos, caseros o de campo", antigua tradición en la Provincia de Corrientes, está implementada exclusivamente como un Sistema Agroalimentario Localizado, que se desarrolla en la mayoría de los establecimientos ganaderos como recurso económico complementario. El queso artesanal se elabora usando metodologías rudimentarias que se transmiten familiarmente en forma oral. Como materia prima se utiliza leche entera cruda de vaca y agente coagulante artesanal. La comercialización se realiza por circuitos informales, exhibiéndolos para la venta conjuntamente con diversos productos de granja en mercados fruti-hortícolas y a la vera de los caminos (Vasek, 2008).

Por otra parte, las condiciones agroecológicas de la región del nordeste argentino (NEA), resultan ideales para algunos cultivos rústicos y nutritivos que pueden utilizarse para la producción ganadera como la caña de azúcar (Saccharum officinarum L.). Su uso en la alimentación de diversas categorías de animales, es muy difundido entre los agricultores familiares ya que presentan un buen rendimiento en la producción de carne y leche cuando se alimentan con este voluminoso. Durante décadas se ha estudiado la caña de azúcar en la alimentación de rumiantes, debido a su alta productividad combinada con el mejor valor energético presentado en invierno, época de escasa disponibilidad de forraje en el nordeste.

El uso de sustancias alcalinizantes como la cal hidratada, sirve para mejorar la digestibilidad y el consumo de alimentos fibrosos, siempre con el objetivo de mejorar su valor nutricional, como voluminosos para rumiantes (Silveira Rabelo, 2011). La mejora del valor nutricional se produce por la ruptura de las fracciones de la pared celular a través de la hidrólisis alcalina (Klopfenstein, 1980), y el aumento de la estabilidad aeróbica de la caña de azúcar, potenciando la producción animal debido a que los microorganismos ruminales hacen un mejor uso de la energía contenida en este alimento.

La caña de azúcar tradicionalmente se corta, pica y se entrega diariamente al animal. Sin embargo, en grandes cantidades, esta técnica se convierte en el mayor obstáculo para el uso de forrajes porque requiere de mano de obra diaria para los cortes, molienda y transporte. La caña de azúcar mezclada con cal (óxido de Calcio) es un método de conservación de forrajes que en esta experiencia nos permitió almacenar el alimento durante 7 días, obteniendo un mejor control en la logística del personal asignado a alimentación y una reducción de los costos asociados con el corte, transporte y la desintegración, especialmente los costos de horas extras durante feriados y fines de semana (Pina et al., 2011) o simplemente aliviar las tareas de la mano de obra familiar.

Desde la Facultad de Ciencias Agrarias de la Universidad Nacional del Nordeste, se trabaja en proyectos de extensión "UNNE en el Medio" relacionados a este tema desde 2015. Esta transferencia de conocimientos y tecnologías se realiza para satisfacer una demanda específica, la participación activa y conjunta de los demandantes y los técnicos. Pero además es importante para nosotros la intervención de estudiantes avanzados de la carrera de Ingeniería Agronómica, dado que es una instancia de aprendizaje y contacto con la realidad productiva, fundamental para su desempeño profesional. Es por ello que en el marco de este ensayo también se desarrolló un Trabajo Final de Graduación para acceder al título de Ingeniero Agrónomo y numerosos estudiantes han aportado a lo largo de estos años a la concreción de los sucesivos proyectos.

Teniendo en consideración los materiales forrajeros regionales de alta disponibilidad y capaces de aportar nutrientes a la dieta animal a bajo costo, se optó por la metodología de Productor Demostrador. Los objetivos de este trabajo fueron que el productor aplique la técnica de conservación de Caña de azúcar picada con agregado de cal (oxido de calcio) y que evalúe la utilización de caña de azúcar picada sin y con agregado de cal en términos de consumo y producción de leche. 


\section{Localización de la experiencia}

La localidad de Santa Ana $\left(27^{\circ} 27^{\prime} 19.08^{\prime \prime}\right.$ S, 58 $39^{\prime} 16.92^{\prime \prime}$ E) está ubicada la noroeste de la provincia de Corrientes, a $15 \mathrm{~km}$ de la Capital provincial, en el departamento San Cosme .

Se trata de una zona de relieve plano con escasa pendiente hacia el sur, dominada por áreas deprimidas de deficiente drenaje (bañados, esteros y cañadas), alternando con lomadas de textura arenosa y baja fertilidad. Los suelos son en su mayoría ácidos, de franco arenosos a franco-arcillo-limosos y con drenaje imperfecto. Predominan las especies típicas del parque chaqueño, como el algarrobo, quebracho blanco y palma caranday. El tapiz natural predominante es de tipo pajonal con especies de mediana a baja calidad. Con sobresaliente presencia de pajonales de paja colorada (Andropogon lateralis) y paja amarilla (Sorghastrum setosum), acompañados por hidrófilas como Leersia sp y Eleocharis sp. (Acosta, 2009).

\section{Descripción de productor}

El Sr. Héctor R. es un productor que hace 30 años trabaja en su establecimiento junto con su familia representada por tres generaciones. Las tareas diarias son realizadas por dos personas. Cuenta con una vivienda y un predio de 60 hectáreas arrendadas, en donde llevan adelante la producción ganadera de cría para la venta de terneros. Tiene 40 vacas con un porcentaje de destete de 50\% y sin estacionamiento del servicio. Durante los primeros meses de lactancia se ordeñan las vacas, con el ternero al pie, una vez al día para elaborar quesos. La alimentación de los animales depende de los pastizales y pajonales mencionados anteriormente. Dispone de $2000 \mathrm{~m}^{2}$ de caña de azúcar que utiliza para suplementar a los animales durante el invierno. Para ello una persona debe realizar diariamente el corte, picado y suministro. Este, como muchos otros productores familiares de la zona desarrolla las actividades económicas típicas de la zona, como son la producción de leche, queso criollo, pollos, huevos, cerdos, conejos, huerta para autoconsumo y venta de los excedentes .

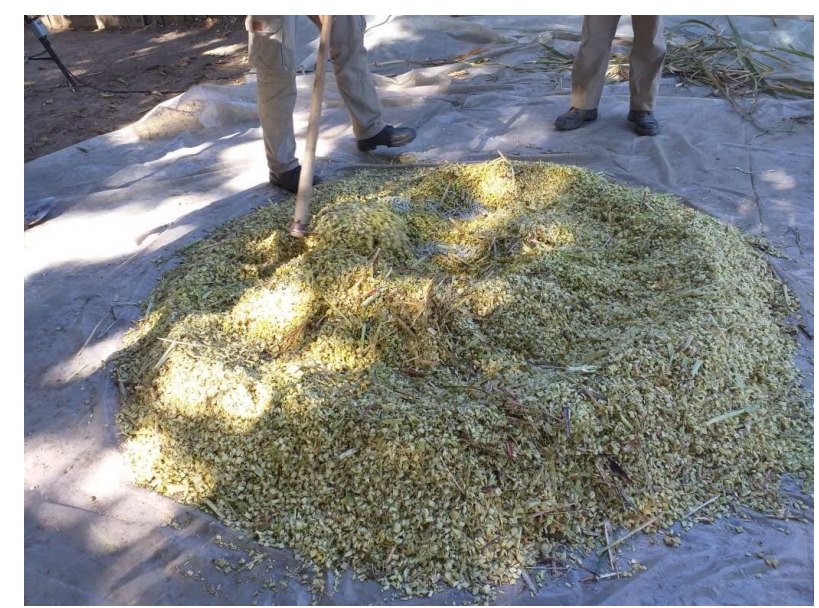

Figura 1. Mezcla de Caña de azúcar picada y cal.

De las vacas en ordeñe, el productor seleccionó tres a las que se suministró diariamente $2 \mathrm{~kg}$ de la mezcla de caña de azúcar tratada, correspondientes al 0,6\% del PV. Se compararon con otras tres que solo recibieron caña de azúcar picada sin cal.

Antes de iniciar la alimentación con la caña de azúcar las vacas solo pastoreaban campo natural. Las evaluaciones se realizaron durante tres semanas, en la primera solo se llevaron registros de la lectura del comedero para establecer el consumo de los animales. Pasado ese período también se midió la producción de leche por día y por animal.

Por otra parte, en el momento de la confección de la mezcla se tomaron muestras para medir en laboratorio el $\mathrm{pH}$ del material y establecer sus cambios diariamente. 


\section{RESULTADOS}

\section{Lectura de comederos}

El productor construyó los comederos con postes y tela plastillera de manera tal que cada animal tenga acceso a 1 metro de comedero. Mediante la lectura de comederos se pudo detectar que durante los dos primeros días de suministro de la caña con cal, los animales consumieron solo un 80\%. Los días siguientes consumieron la totalidad, hasta el día 6 que volvieron a dejar un 20\% en los comederos .

El rechazo inicial se puede atribuir a que estaban reconociendo el alimento y el de los últimos días a un deterioro en la palatabilidad del mismo.

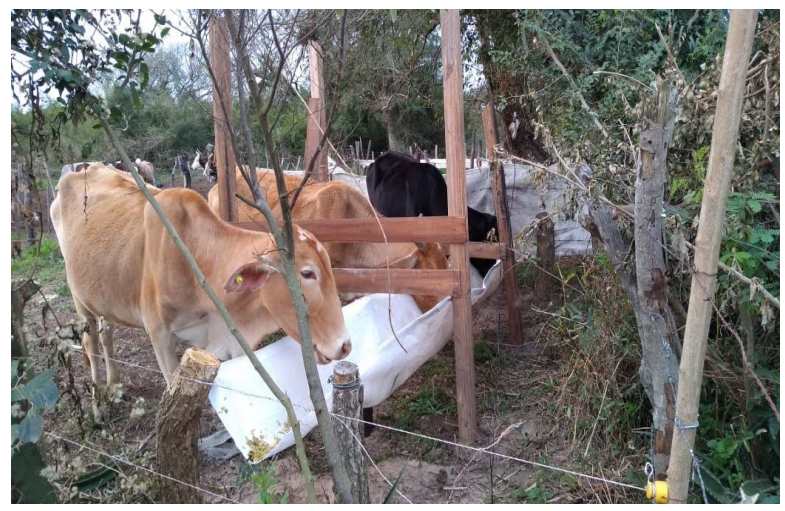

Figura 2. Vacas comiendo Caña picada con cal. Ensayo en el predio del productor demostrador. en Santa Ana, Corrientes

\section{Producción de leche}

La producción de leche diaria por cabeza aumentó aún con el suministro de caña de azúcar sin cal (Figura 3). Sin embargo con la adición de cal el incremento fue mayor, superando a la de caña de azúcar sola en un $8 \%$ y $32 \%$ en la segunda y tercer semana respectivamente.

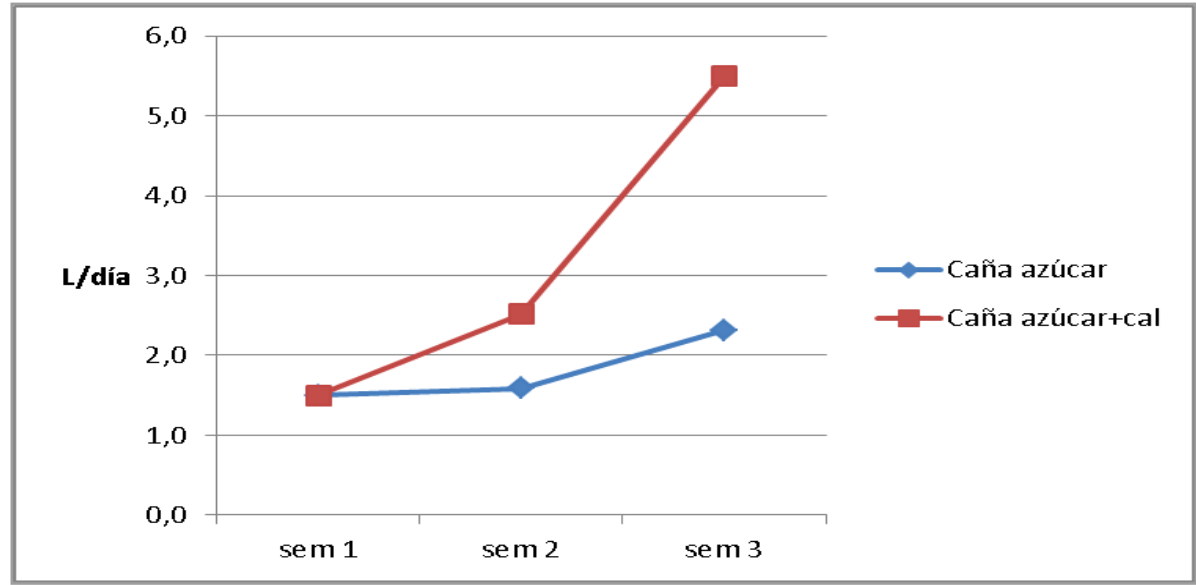

Figura 3. Producción de leche, 1/día, promedio de tres vacas durante las semanas del ensayo.

pH

Las variaciones diarias de $\mathrm{pH}$ en la caña con y sin el agregado de cal se muestran en la Figura 4.

El pH de la caña picada fue disminuyendo hasta estabilizarse a las 72 horas en ambos tratamientos. Aunque este hecho ocurre naturalmente por la acción de los microorganismos que consumen carbohidratos solubles causando la acidificación de la masa, la diferencia sería que el tratamiento sin cal tendría menor valor nutricional.

El hecho de que la hidrólisis proporcione más estabilidad aeróbica a la caña de azúcar picada se asocia con el tiempo que puede permanecer en el comedero sin fermentaciones no deseadas. En consecuencia los alimentos se pueden suministrar en cantidades mayores por comida, pero con menos frecuencia, reduciendo así los costos de operación que implican mano de obra (Silveira Ravelo, 2011). 


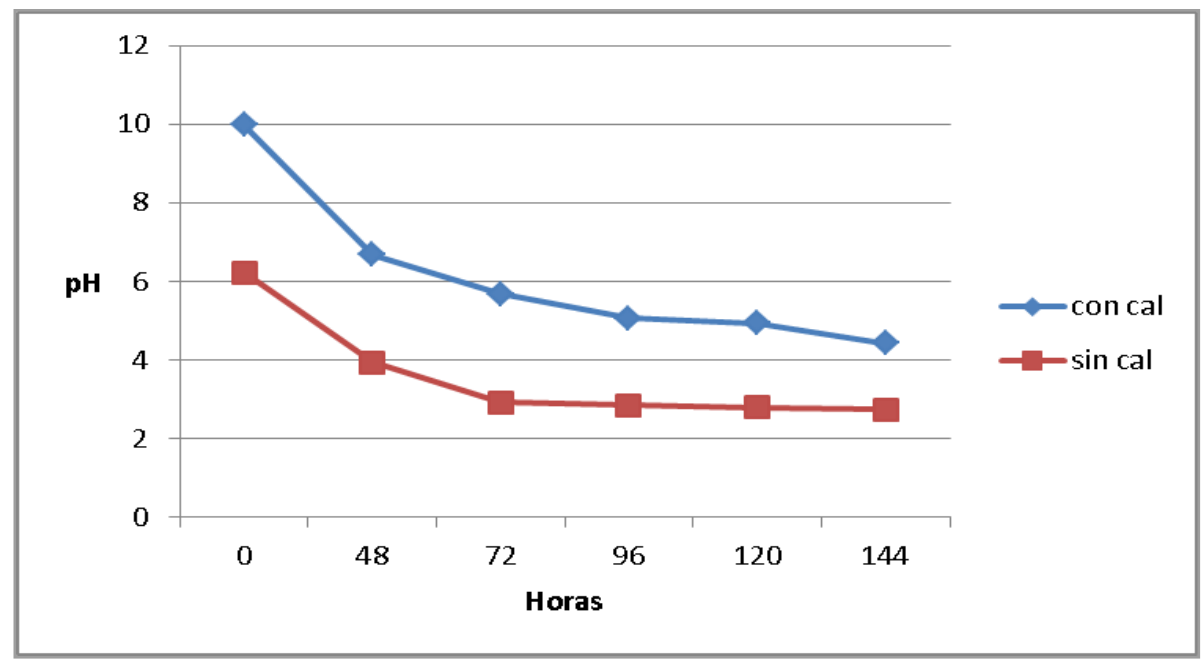

Figura 4. pH de la caña de azúcar picada durante las semanas del ensayo.

\section{CONCLUSIÓN}

Los resultados obtenidos en el ensayo realizado con el productor son de gran valor técnico y se espera repetirlos en campañas siguientes.

El productor se involucró en todas las decisiones para el desarrollo del ensayo. También en el registro diario del suministro de la caña y la producción de leche. Una vez analizados los resultados evaluó positivamente la experiencia. El mismo se mostró satisfecho ya que no solo incrementó la producción de leche diaria sino que también pudo organizar mejor el tiempo dedicado a la alimentación de los animales. Por ello, podrá compartir una técnica evaluada y aplicada por él a sus pares con lo que se espera la difusión y transferencia tenga mayor impacto.

\section{REFERENCIAS}

Acosta, F., Giménez, L., Richieri, C. y Calvi, M. (2009). Zonas AgroEconómicas Homogéneas. Corrientes. INTA. Estudios socioeconómicos de la sustentabilidad de los sistemas de producción y recursos naturales. 8: $75 \mathrm{p}$.

Klopfenstein, T. (1980). Increasing the nutritive value of crop residues by chemical treatments. In: Silveira Rabelo, C. H. et all, (2011). Estabilidade Aeróbia De Cana-De-Açúcar Innatura Hidrolisada Com Cal Virgem. Ci. Anim. Bras., Goiânia. 1 y 2 (2) : 257-265.

Pina, D.D.P, Filho, S.D.C, Tedeschi, O.L, Martins, A.B, Gomez, J.A.A, Valadarez, R.F.D., Souza, K.N.D.P y Fonseca, M.A. (2011). Los niveles de inclusión y tiempo de exposición de la caña de azúcar en óxido de calcio sobre los parámetros digestivos y el desempeño de novillas Nellore. R. Bras. Zootec. (Online). 40 (3) : 648-656.

Silveira Rabelo, C.H., Vilela De Rezende, A., Silveira Rabelo, F.H., Alves Nogueira, D., Ferreira Elias, R. y Nogueira Achcar De Faria Júnior, D. (2011). Estabilidade Aeróbia De Cana-De-Açúcar Innatura Hidrolisada Com Cal Virgem. Ci. Anim. Bras., Goiânia, 1 y 2 (2) : 257-265. DOI: 10.521 6/cab.v1 2i2.8525

SENASA. (2011). Estadísticas: Caracterización de la producción ganadera. Elaboración DGB - MAGyP. http:// www.senasa.gob.ar/cadena-animal/bovinos-y-bubalinos/informacion/informes-y-estadisticas.

Vasek, O.M., Cardozo, M.C., Fusco, A. J. V. (2008). Producción Artesanal De Quesos. Sistema De Transformación Agroalimentario en la Región Correntina (ARGENTINA). IV Congreso Internacional de la Red SIAL. 